\title{
Mapeando la investigación sobre COVID-19 en Argentina: análisis bibliométrico a 6 meses
} del primer caso reportado

\author{
Mapping research on COVID-19 in Argentina: bibliometric analysis 6 months after the first \\ reported case
}

\section{Mapeamento da pesquisa sobre COVID-19 na Argentina: análise bibliométrica 6 meses após o primeiro caso relatado}

\author{
Javier González Argote ${ }^{1}$.
}

La pandemia por COVID-19, ha generado una movilización de la comunidad cientifica a nivel mundial y una emergencia de las investigaciones sobre el tema. Este artículo analiza basado en las publicaciones científicas de la base de datos Scopus el comportamiento de la producción científica sobre COVID-19 por autores argentinos en los 6 primeros meses desde la detección del primer caso en el país. Se analiza la cantidad de publicaciones, colaboración científica, producción a nivel jurisdiccional e institucional y análisis cualitativo.

\section{Conceptos clave}

Que se sabe del tema: Resulta llamativo que en los estudios de evaluación de la ciencia sobre el COVID-19, no figura Argentina con artículos científicos publicados.

Que aporta este artículo: El presente artículo describe la cantidad de artículos publicados, instituciones donde realizaron, la colaboración nacional e internacional que tuvieron los artículos sobre COVID-19 publicados en los primeros 6 meses luego de la pandemia donde participó al menos un autor argentino; hallazgos que nos permiten inferir que en Argentina existió una respuesta efectiva de la comunidad científica ante la crisis sanitaria y social sin precedentes que impuso el COVID-19, orientadas a la resolución de problemas locales, y particularmente contribuir desde la ciencia a mitigar su propagación e impacto en el sistema sanitario.

1- Universidad Maimónides, Facultad de Ciencias de la Salud, Carrera de Licenciatura en Enfermería. Buenos Aires; Argentina. ORCID: http://orcid.org/0000-0003-0257-1176 . E-mail de contacto: jargote27@gmail.com

\section{Resumen:}

Introducción: El brote de COVID-19 ha causado una gran amenaza a la comunidad internacional y ha planteado importantes preocupaciones de salud pública. El objetivo de este trabajo fue describir los patrones de comunicación científica de Argentina sobre COVID-19 en la base de datos Scopus. Método: Se realizó un estudio bibliométrico. Se recuperaron artículos según la temática utilizando los campos del título, resumen y palabras clave en la base de datos Scopus. Resultados: Se recuperaron 229 documentos, donde el $69,9 \%$ eran documentos citables (51,5\% de originales), el $76,4 \%$ en idioma inglés. El liderazgo científico de Argentina en los documentos publicados fue de un $44,1 \%$. Se encontró colaboración nacional en el $38 \%$ de los documentos, en el $16,6 \%$ colaboración entre instituciones de jurisdicciones diferentes y el $59,8 \%$ colaboración internacional. Conclusiones: Los indicadores de productividad e internacionalización encontrados en el presente estudio representan la respuesta de la comunidad científica argentina en la fase inicial desde la esfera de la producción del conocimiento. Los resultados obtenidos podrían interpretarse como una respuesta efectiva ante la crisis sanitaria y social sin precedentes que impuso el COVID-19.

Palabras clave: infecciones por coronavirus; Argentina; gestión del conocimiento; gestión del conocimiento para la investigación en salud.

\section{Abstract:}

Introduction: The COVID-19 outbreak has caused a major threat to the international community and raised significant public health concerns. The objective of this work was to describe the scientific communication patterns of Argentina on COVID-19 in the Scopus database. Method: A bibliometric study was carried out. Articles were retrieved according to subject matter using the title, abstract and keywords fields in the Scopus database. Results: 229 documents were retrieved, where $69.9 \%$ were citable documents (51.5\% originals), $76.4 \%$ in English. The scientific leadership of Argentina in the published documents was $44.1 \%$. National collaboration was found in $38 \%$ of the documents, in $16.6 \%$ collaboration between institutions from different jurisdictions, and $59.8 \%$ international collaboration. Conclusions: The productivity and internationalization indicators found in this study represent the response of the Argentine scientific community in the initial phase from the sphere of knowledge production. The results obtained could be interpreted as an effective response to the unprecedented health and social crisis imposed by COVID-19.

Keywords: coronavirus infections; Argentina; knowledge management; knowledge management for health research

\section{Resumo:}

Introdução: O surto de COVID-19 causou uma grande ameaça à comunidade internacional e levantou questões de saúde pública significativas. O objetivo deste trabalho foi descrever os padrões de comunicação científica da Argentina sobre o COVID-19 na base de dados Scopus. Método: Foi realizado um estudo bibliométrico. Os artigos foram recuperados de acordo com a temática nos campos título, resumo e palavras-chave da base de dados Scopus. Resultados: foram recuperados 229 documentos, dos quais $69,9 \%$ eram documentos citáveis (51,5\% originais), $76,4 \%$ em inglês. A liderança científica da Argentina nos documentos publicados foi de $44,1 \%$. Colaboração nacional foi encontrada em $38 \%$ dos documentos, em $16,6 \%$ de colaboração entre instituições de diferentes jurisdições e em $59,8 \%$ de colaboração internacional. Conclusões: Os indicadores de produtividade e internacionalização encontrados neste estudo representam a resposta da comunidade científica argentina na fase inicial da produção de conhecimento. Os resultados obtidos podem ser interpretados como uma resposta eficaz à crise social e de saúde sem precedentes imposta pelo COVID-19.

Palavras-chave: infecções por coronavirus; Argentina; gestão do conhecimento; gestão do conhecimento para a pesquisa em saúde. 


\section{Introducción}

El brote de COVID-19 ha causado una gran amenaza a la comunidad internacional $y$ ha planteado importantes preocupaciones de salud pública. La amplia propagación de esta enfermedad, junto con su alta tasa de infectividad, ha incitado una demanda mundial de investigación relevante que pueda ayudar a describir las características clínicas y patogénicas de esta enfermedad ${ }^{(1)}$

El 3 de marzo se detectó el primer caso en el país, luego de varias falsas alarmas y numerosos casos sospechosos que arrojaron resultados negativos ${ }^{(2)}$. El 19 de marzo de 2020 en una conferencia de prensa por el presidente Alberto Fernández se decretó la cuarentena en Argentina, cuya denominación oficial es Aislamiento Social, Preventivo y Obligatorio (ASPO). Esta fue decretada a nivel nacional por decisión del Gobierno de la República Argentina a elección unánime mediante el Decreto Nacional de Urgencia (DNU) $297 / 2020^{(3)}$.

Podría decirse que el COVID-19 ha sido el foco principal de las instituciones médicas y científicas de todo el mundo durante los últimos meses ${ }^{(1)}$, dado la preocupación a nivel mundial de la actual pandemia que vivimos es un área que cobra cada vez más importancia.

Los resultados individuales de estudios bibliométricos sobre el tema en la base de datos Scopus varían respecto al número de documentos e indicadores a nivel global como los de Chahrour $y$ $\operatorname{cols}^{(1)}$, Lou y $\operatorname{cols}^{(4)}$, O'Brien y $\operatorname{cols}^{(5)}$, Zyoud y Al-Jabi ${ }^{(6)}$, y en el contexto latinoamericano como el de Gregorio-Chaviano y $\operatorname{cols}^{(7)}$, sin embargo resulta llamativo el hecho que en ninguno figura Argentina con producción científica.

Resulta pertinente entonces realizar un análisis bibliométrico que dilucide si realmente la producción científica argentina sobre COVID-19 es baja o nula; a su vez los indicadores de productividad de los conocimientos científicos permitirán visibilizar la investigación y contextualizarla en función de indicadores de salud del país.

El objetivo de este trabajo es describir los patrones de comunicación científica de Argentina sobre COVID-19 en la base de datos Scopus.

\section{Material y Métodos}

Tipo de estudio: Bibliométrico.

Fuente de datos:

Se recuperaron artículos según la temática utilizando los campos del título, resumen y palabras clave en la base de datos Scopus el día 7 de septiembre de 2020. Fue utilizada la base de datos Scopus, considerada la es la más grande base de datos de resúmenes y citas de la literatura revisada por pares, con diversas herramientas de análisis ${ }^{(8)}$; de igual forma análisis bibliométricos anteriores sobre el COVID-19 han utilizado preferencialmente esta base de datos ${ }^{(1,6,9)}$ sobre otras como Web of Science.

\section{Estrategia de búsqueda:}

Se utilizó la función "TITLE-ABS-KEY" que busca la presencia de los términos o frases en el Título, Resumen y Palabras clave de los documentos, por otra parte, se filtraron los documentos donde al menos un autor perteneciera a una institución de Argentina, para lo que se usó la función "AFFILCOUNTRY". Integrando ambos aspectos y tomando como referencia estudios bibliométricos previos sobre el tema se construyó la estrategia de búsqueda: TITLE-ABSKEY("COVID 19" or "2019 novel coronavirus" or "coronavirus 2019" or "coronavirus disease 2019" or "2019-novel CoV" or "2019 ncov" or "COVID 2019" or "COVID19" or "corona virus 2019" or "nCoV2019" or "nCoV2019" or "nCoV 2019" or "2019-ncov" or "COVID-19" or "Severe acute respiratory syndrome coronavirus 2" or "SARSCoV-2") AND AFFILCOUNTRY(Argentina)

\section{Análisis bibliométrico:}

Se recuperaron 229 documentos, que fueron descargados a una base de datos en formato RIS desde Scopus. Luego fueron procesados con diversos programas (Bibexel, Excel y Access) para obtener los indicadores bibliométricos: número de documentos (Ndoc), tipos de documentos, provincia (jurisdicción)/país, institución, colaboración (nacional $(\mathrm{CN})$, internacional $(\mathrm{Cl})$ y interjurisdiccional) y categoría temática (según el "All Science Journal Classification Codes - ASJC").

Visualización de redes:

Las matrices de co-ocurrencia para el análisis de las redes sociales entre los países y términos fueron desarrollados con los programas Bibexel y VOSviewer 1.6.15 (https://www.vosviewer.com/) fueron utilizados para visualizar las relaciones entre dichas redes. Se realizó normalización de los campos de país y las palabras clave, específicamente en esta última se tomaron aquellos términos con una frecuencia de aparición mayor o igual a 100.

Análisis estadístico:

Se emplearon técnicas de la estadística descriptiva: números absolutos y porcientos y estadística inferencial. Los resultados de las variables se procesaron mediante el programa Microsoft Excel y el paquete estadístico InfoStat.

Para el establecimiento de la relación entre las variables se determinó el coeficiente de correlación de Spearman ( $r$ ). Las diferencias se consideraron significativas con valores de $p$ menores que 0,05 .

Fuentes Primarias:

Las bases de datos y fuentes primarias utilizadas fueron depositadas en el repositorio de datos Zenodo (http://doi.org/10.5281/zenodo.4019994).

\section{Resultados}

Se recuperaron un total de 229 documentos, los que según su tipología se distribuyen en: Originales $(n=118 ; \%=51,5 \%)$, Revisiones $(37 ; 16,2 \%)$, Cartas $(31 ; 13,5 \%)$, Notas $(20 ; 8,7 \%)$, Editoriales $(18 ; 7,9 \%)$; Originales breve $(4 ; 1,7 \%)$ y Artículo de conferencia (1;0,4\%).

En relación al idioma en que se publicaron los artículos el 175 $(76,4 \%)$ fueron en idioma inglés, $50(21,8 \%)$ en español, $3(1,3 \%)$ en francés y $1(0,4 \%)$ en portugués.

En la Figura 1 se muestran los documentos publicados según jurisdicción (Figura 1-A) y los casos confirmados de COVID-19 acumulados al 7 de septiembre de 2020 según jurisdicción en Argentina (Figura 1-B). Al analizar el coeficiente de correlación entre estas dos variables se encontró una correlación positiva ( $r=$ 0,$9657 ; \mathrm{P}<0,0001 ;$ IC $95 \%=0,9212$ a 0,9853). 


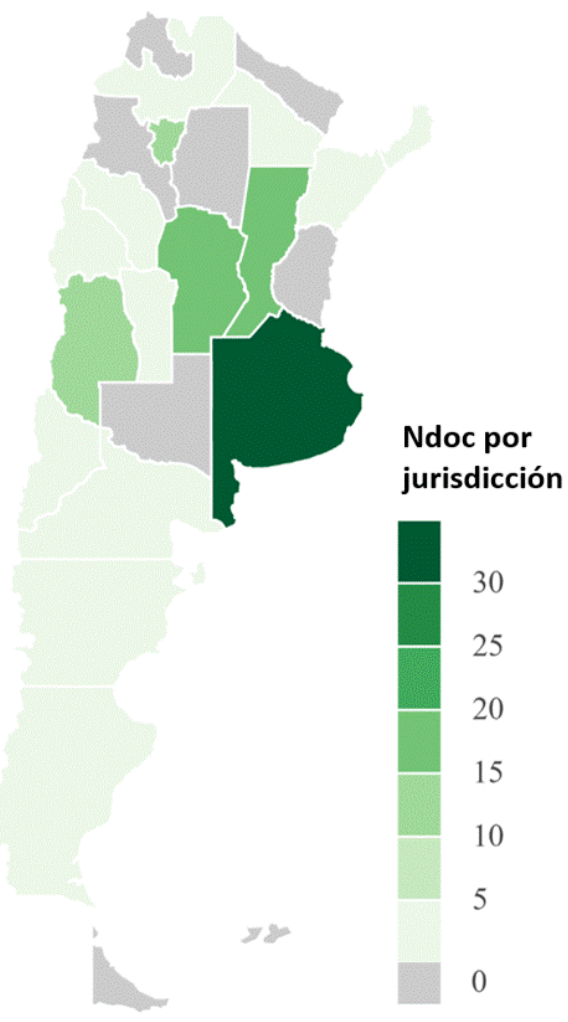

A

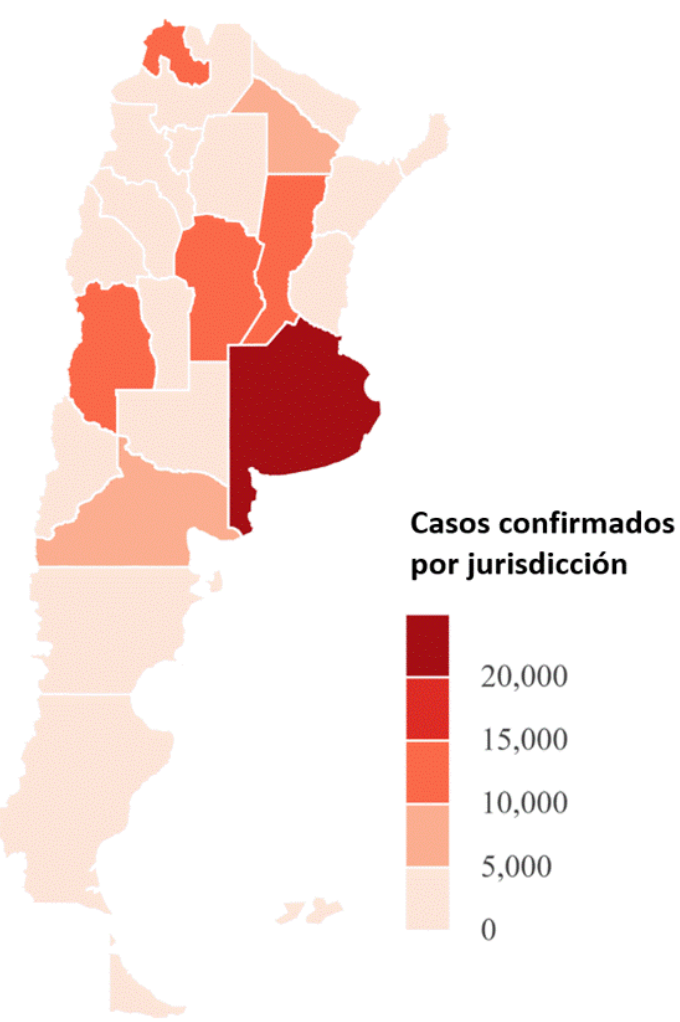

B

El liderazgo científico de Argentina en los documentos publicados fue de un $44,1 \%$ (101), este indicador se mide cuantificando el número de documentos donde el autor de correspondencia pertenecía a una institución de este país y en el caso que este campo no estuviera presente se toma la institución del primer autor. En 87 artículos (38,0\%) hubo colaboración nacional (entre dos instituciones argentinas independientemente de la jurisdicción), en el $16,6 \%$ (38) de los artículos hubo colaboración entre instituciones de jurisdicciones diferentes y el 59,8\% (137) tuvo colaboración internacional; de ellos en el $16,1 \%$ hubo liderazgo científico de Argentina.

En la Figura 2 se muestra las redes de colaboración internacional encontrada en los artículos publicados sobre la temática.

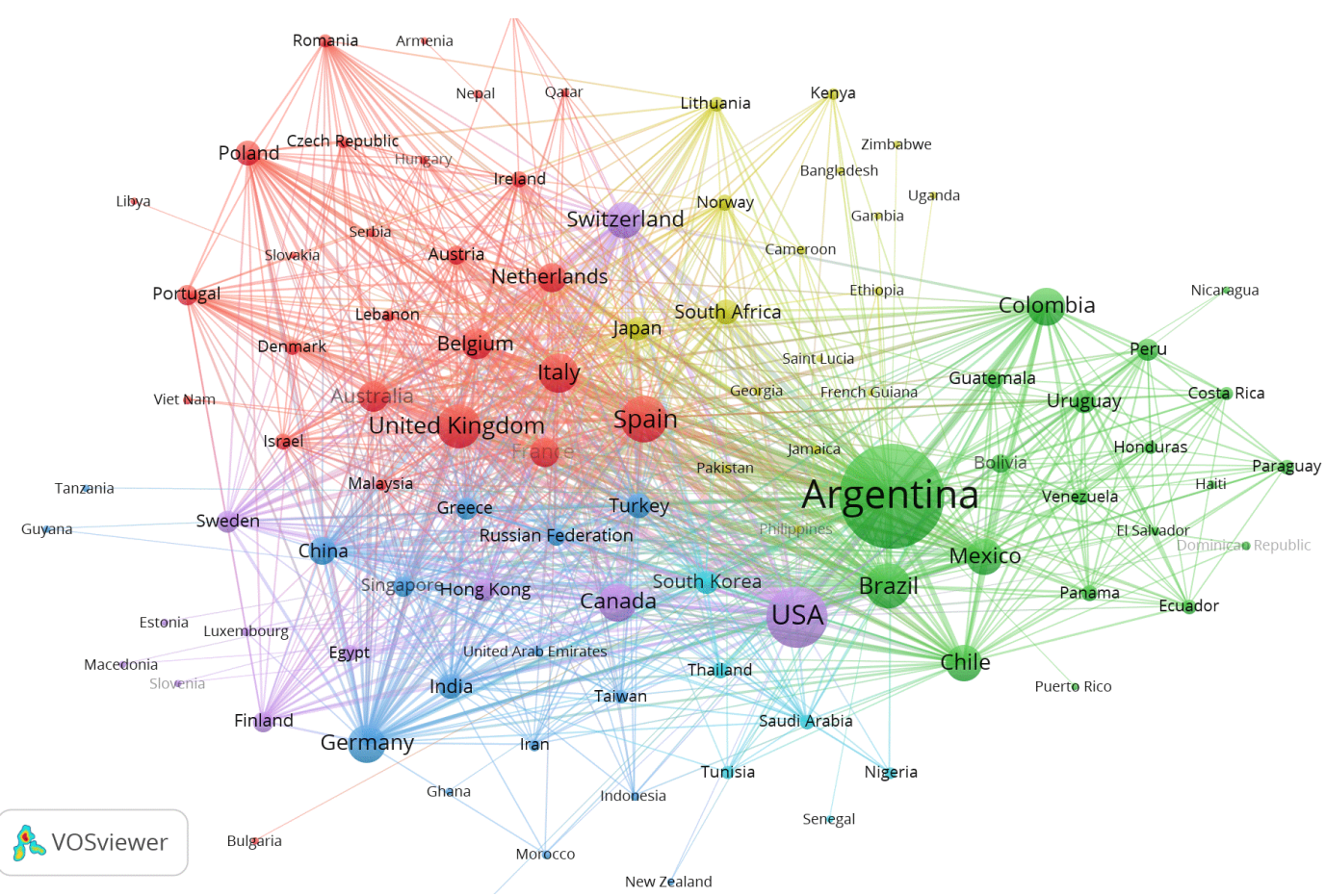

Figura $\mathbf{N}^{\circ}$ 2. Red de colaboración internacional de los artículos publicados sobre COVID-19 en Argentina. 
En la Tabla 1 se muestran las 21 instituciones argentinas que publicaron al menos 4 documentos sobre el COVID-19, y sus indicadores de colaboración nacional e internacional (El listado completo del resto de las instituciones argentinas se puede ver en el siguiente enlace http://doi.org/10.5281/zenodo.4019994).

Tabla $N^{\circ} 1$ : Distribución de la producción científica según instituciones argentinas.

\begin{tabular}{|c|c|c|c|c|}
\hline Ranking & Institución & Ndoc & $\% \mathrm{CI}$ & $\% \mathrm{CN}$ \\
\hline 1 & Universidad de Buenos Aires & 37 & 43,2 & 48,6 \\
\hline 2 & Hospital Italiano de Buenos Aires & 28 & 42,9 & 53,6 \\
\hline 3 & $\begin{array}{l}\text { Consejo Nacional de Investigaciones Científicas y Técnicas } \\
\text { (CONICET) }\end{array}$ & 13 & 38,5 & 92,3 \\
\hline 4 & Instituto Cardiovascular de Buenos Aires (ICBA) & 9 & 44,4 & 77,8 \\
\hline 5 & Hospital Alemán de Buenos Aires & 8 & 75 & 50 \\
\hline 6 & FLENI & 6 & 50 & 50 \\
\hline 6 & Complejo Médico de la Policía Federal Argentina Churruca Visca & 6 & 100 & 33,3 \\
\hline 6 & Hospital General de Agudos Dr. Juan A. Fernández & 6 & 83 & 50 \\
\hline 6 & Hospital Nacional de Pediatría Juan P. Garrahan & 6 & 100 & 50 \\
\hline 7 & Universidad Nacional de Córdoba & 5 & 20 & 60 \\
\hline 7 & Universidad Nacional de la Plata & 5 & 40 & 20 \\
\hline 7 & CEMIC & 5 & 60 & 100 \\
\hline 7 & Universidad Nacional de Cuyo & 5 & 60 & 60 \\
\hline 7 & Hospital de Alta Complejidad El Cruce - Néstor Kirchner & 5 & 60 & 80 \\
\hline 7 & Hospital Británico de Buenos Aires & 5 & 80 & 60 \\
\hline 7 & Sanatorio Güemes & 5 & 40 & 80 \\
\hline 7 & Universidad Austral & 5 & 40 & 60 \\
\hline 7 & Centro Privado de Cardiología (Tucumán) & 5 & 80 & 20 \\
\hline 8 & Universidad Nacional del Litoral & 4 & 25 & 75 \\
\hline 8 & Universidad Nacional de Rosario & 4 & 50 & 100 \\
\hline 8 & Universidad Nacional de Tucumán & 4 & 50 & 25 \\
\hline
\end{tabular}

Al analizar la matriz de co-ocurrencia de términos (Figura 3) y categorías temáticas (Figura 4) por medio de los mapas bibliométricos, fueron considerados aquellos donde hubo co- ocurrencia para términos de 100 más veces, y para las categorías 2 o más veces. 


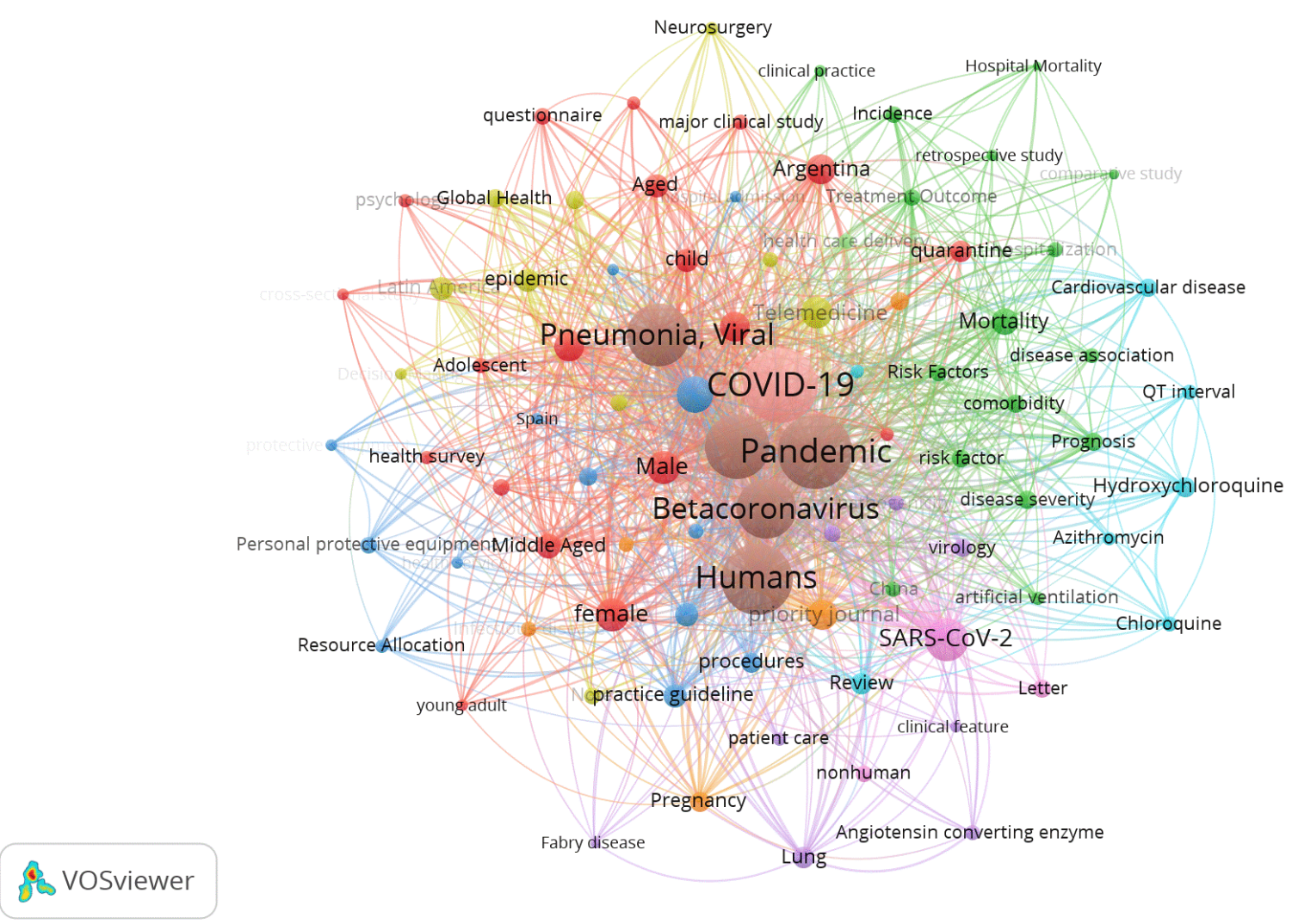

Figura $\mathbf{N}^{\circ}$ 3. Mapa de co-ocurrencia de términos de los artículos publicados sobre COVID-19 en Argentina.

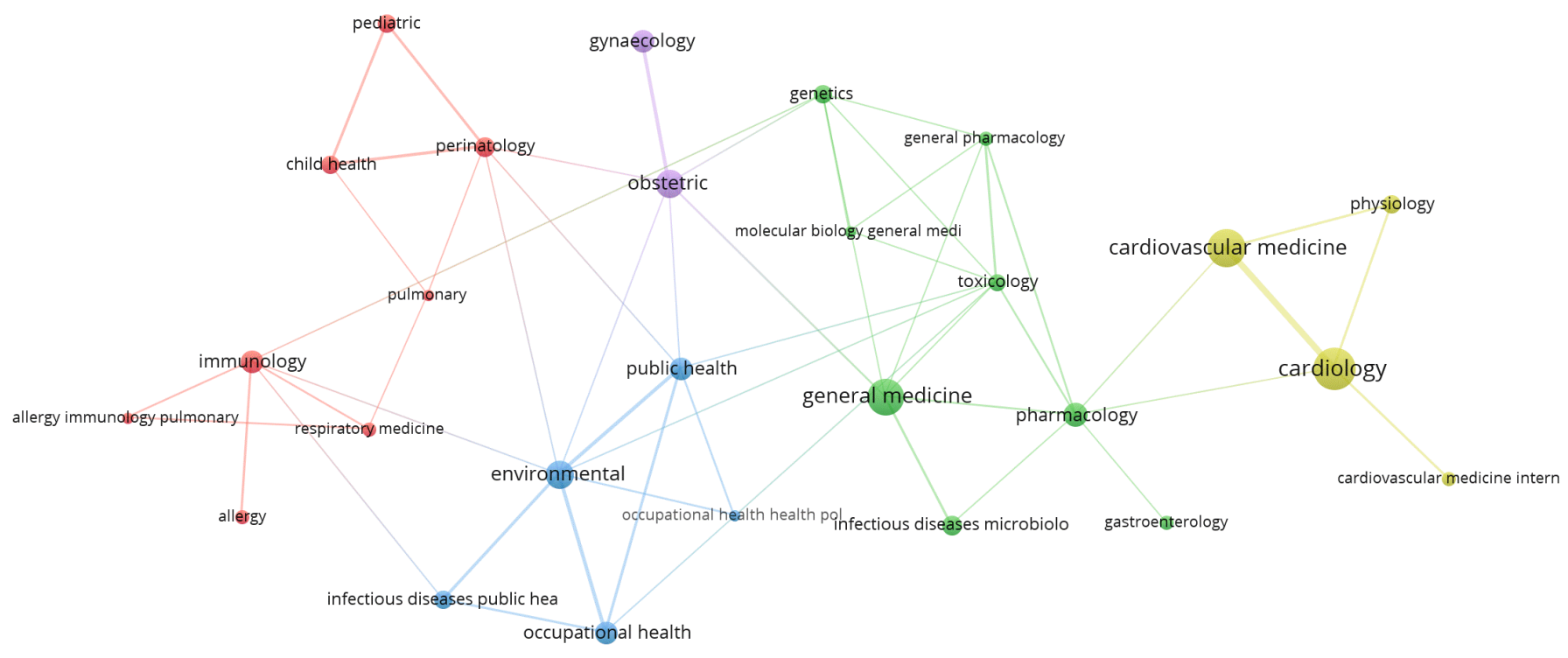

禹 Vosviewer

Figura $\mathrm{N}^{\circ}$ 4. Mapa de co-ocurrencia de categorías de las publicaciones sobre COVID-19 en Argentina.

\section{Discusión}

El estudio actual demuestra un número considerable en las investigaciones sobre COVID-19, si se tiene en cuenta el corto período de tiempo desde que se detectó el primer caso en
Argentina, podemos afirmar que en base se evidencia una rápida respuesta de la comunidad científica, si bien es cierto que esta solo representa una parte de la producción científica real que pudiera existir, no obstante al ser Scopus una base de datos con amplia cobertura y altos estándares de calidad en la selección de los recursos que indexa sirve como un excelente termómetro científico. 
Dada la heterogeneidad temporal de los estudios sobre COVID-19 en la base de datos Scopus tanto a nivel mundial ${ }^{(1,4-6)}$, y en Latinoamérica $^{(7)}$, este estudio se centró fundamentalmente en caracterizar la producción científica nacional más que en contextualizarla en el ámbito internacional. Si bien existen aproximaciones a nivel mundial es necesario darle más tiempo a la comunidad científica a publicar sus investigaciones, dada las diferencias en los patrones de comunicación en las distintas áreas de la ciencia. Sin embargo, este estudio arroja resultados que subrayan las conclusiones obtenidas en gran parte de los trabajos previos de este campo a nivel mundial.

Un hallazgo a destacar fue que el 69,9\% (160) de los documentos eran documentos citables (considerándose en esta categoría los artículos originales, revisiones, artículos de conferencias y originales breves) lo que representa una fortaleza ya que suponen resultados de investigaciones científicas relevantes, son el vehículo más frecuentemente utilizado para comunicar nuevos conocimientos y generalmente el impacto de este tipo de artículo es superior a la media.

Chahrour y cols encontraron un $77 \%$ de originalidad y afirman que para un tema de suma importancia médica, se puede esperar tener un mayor número de publicaciones originales ${ }^{(1)}$.

Aunar esfuerzos para enfrentar el avance del virus se ha convertido más que un eslogan, en una realidad imprescindible en tiempos de pandemia de COVID-19, ello ha involucrado todas las esferas de la sociedad.

Estudios como los de Juárez y Barrere reportan que la cooperación nacional en Argentina predominó entre institutos científicos y unidades académicas con un $47.66 \%$, mientras que la cooperación internacional se encontró en el $59.09 \%$ de los artículos analizados ${ }^{(10)}$. Estos resultados contrastan con los valores encontrados en este estudio.

A partir de los indicadores de colaboración tanto nacional como internacional y los mapas bibliométricos se respalda el hecho que existen sólidas redes de colaboración tanto entre instituciones argentinas como con instituciones extranjeras, que en este momento de crisis supieron responder de forma efectiva en la producción de conocimiento a pesar de las limitaciones que impuso la pandemia.

La colaboración es elemento muy tratado en los estudios bibliométricos y caracteriza un dominio científico, los que sin dudas lleva a generar un aumento del impacto y visibilidad de los artículos, comparado con aquellos que son escritos solo por autores de una misma institución.

Este estudio confirma que no es un hecho fortuito que Estados Unidos y España sean los países con mayor colaboración internacional, como bien han revelado Zyoud y Al-Jabi el papel de liderazgo desempeñado por Estados Unidos, China, Italia y el Reino Unido en la investigación de COVID-19, y afirman que una posible razón de estos hallazgos puede atribuirse a la alta prevalencia de COVID-19 en los países que presenciaron el primer brote. Estados Unidos tiende a tener condiciones superiores para la investigación médica básica o los ensayos experimentales, que incluyen fondos y recursos suficientes, equipo avanzado e investigadores capacitados $^{(6)}$.

El hallazgo más sorprendente de los indicadores de productividad fue que en el $44,1 \%$ de los documentos el líder de la investigación fuera un autor argentino, y aún más que el $16,1 \%$ de los artículos con colaboración internacional fueran liderados por autores nacionales.

Miguel y cols en un análisis de las dimensiones nacionales e internacionales de la investigación científica argentina con visibilidad en Scopus encontraron que el liderazgo científico en la producción científica argentina es alto $(74 \%)$; y cuando se analiza el liderazgo en la producción en colaboración internacional se observa claramente que los autores argentinos tienen un mayor protagonismo en las producciones que ronda el $40 \%{ }^{(11)}$.

Zacca-González plantea que diferenciar el conocimiento científico de excelencia producido con liderazgo de aquel en el que la nación o la institución participa, pero con el liderazgo de otros, permite reconocer mejor las más genuinas capacidades científicas de un país $^{(12)}$.
Como es de espera las provincias con mayor producción científica (Buenos Aires, Ciudad de Buenos Aires, Santa Fe, Córdoba, Tucumán y Mendoza), son precisamente las que mayor invención en $1+D^{(13)}$, es esperable que su capacidad tecnológica sea mayor, así como la vinculación y/o desarrollo de nuevos proyectos.

El $58 \%$ de los documentos pertenecen a la provincia de Buenos Aires, que reúne poco más del $30 \%$ de la inversión pública y privada total en I+D de Argentina. Su magnitud es tal que, si fuese un país, podría ocupar el cuarto lugar en inversión en este rubro, después de Brasil, México y Argentina; es el distrito que produce la mayor cantidad de publicaciones científicas y el que tiene mayor número de personas dedicadas a actividades de $I+D^{(14)}$.

La fuerte asociación entre las provincias con mayor número de casos y su mayor productividad, más allá del análisis relativo a la financiación en ciencia, denota la respuesta ante una situación epidemiológica tan compleja e inesperada como es la pandemia de COVID-19 a nivel mundial. Otro elemento a destacar es que son precisamente las provincias con mayor número de casos las que más oportunidades tienen de estudiar tanto las muestras como los individuos enfermos.

Con la finalidad promover Proyectos de $1+D+i$ con objetos de estudio sobre el diagnóstico, el control, la prevención, el tratamiento y el monitoreo, del COVID-19, la Agencia de Promoción de la Investigación, el Desarrollo y la Innovación (Agencia I+D+i) en el marco de la Unidad Coronavirus, financió 64 iniciativas que recibirán una suma máxima en pesos equivalente a 100.000 dólares ${ }^{(15)}$. A pesar de existir una lista pública de los investigadores principales de los proyectos, es muy reciente para evaluar la producción científica derivada de ellos, no obstante, sería interesante a futuro el análisis de su productividad e impacto.

Históricamente el CONICET ha liderado la producción científica argentina en Scopus ${ }^{(16)}$, sin embargo en este caso particular el primer lugar lo ocupó la Universidad de Buenos Aires tanto en indicadores de productividad como colaboración.

Liderados por el Hospital Italiano de Buenos Aires, destacan los centros hospitalarios entre los más productivos, estos resultados posicionan los hospitales como instituciones altamente productivas en el tema, y con colaboración científica. Es importante saber que el hecho que se brinde atención médica a pacientes positivos para COVID-19 no necesariamente significa que ese potencial se utilice para producir ciencia.

Estos hallazgos sustentan la premisa que los hospitales constituyen centros de alto nivel científico técnico y elevada capacidad resolutiva encargada de recuperar y rehabilitar la salud de la población, por ello representa una de las directrices del Ministerio de Salud la investigación como expresión concreta en la solución de los principales determinantes de las enfermedades en función del fortalecimiento los sistemas y servicios de salud.

Al analizar las palabras claves utilizadas en los artículos y sus relaciones a través de grafos (mapa de coocurrencia de términos). Se visualizan 6 núcleos, lo que nos puede aproximar a las principales líneas de investigación; el nodo central incluye los descriptores objeto de estudio (SARS-CoV-2, COVID-19, Coronavirus), asociados a este nodo encontraron los relacionados con ámbito de la salud pública, biología molecular, virología, epidemiología y la farmacología. El análisis de las redes de términos constituye una información valiosa, por un lado, están ajenas a la intervención de un analista y por el otro refleja las redes de relaciones conceptuales.

Las redes de categorías sustentan los resultados encontrados en las palabras claves, destacan las áreas relacionadas con la inmunología, salud pública farmacología y cardiología, lo que está directamente relacionado con los temas que más preocupan a la comunidad internacional: el impacto sobre la atención a pacientes, los sistemas y servicios de salud, las patologías cardiovasculares previas como factor de riesgo y marcador de mal pronóstico de la enfermedad y el desarrollo de un fármaco capaz de generar inmunidad ante el virus.

Zyoud y Al-Jabi en un estudio bibliométrico a nivel global sobre COVID-19 encontraron como temas principales las características clínicas, los hallazgos patológicos y el diseño terapéutico, la preparación de los centros de atención y el control de infecciones, y 
los resultados maternos, perinatales y neonatales, y plantean que estos podrían ser una frontera de investigación en el futuro ${ }^{(6)}$.

\section{Limitaciones y perspectivas futuras}

La primera limitación del estudio es que el análisis se realiza en el contexto de la pandemia que hoy se vive de COVID-19, que a la fecha no ha finalizado en el país, sin embargo, se convierte este artículo en una especie de termómetro de cómo se encuentra la producción científica sobre el tema.

La segunda limitación es el hecho que se utilizara solo la base de datos Scopus, a pesar de su amplia cobertura se convierte también en una limitación, si bien es cierto que la normalización y estandarización de los metadatos que provee sobre todo lo referente a la filiación es superior; no obstante, futuras investigaciones podrían incluir Medline, Web of Science, SciELO, Redalycs y otras, y analizar el dominio sobre el tema con una mayor cobertura y diversidad.

Por otro lado, en este artículo no fueron analizados indicadores de citación dado que es muy reciente para realizar un análisis de este ámbito bibliométrico.

\section{Conclusiones}

Los indicadores de productividad e internacionalización encontrados en el presente estudio representan la respuesta de la comunidad científica argentina en la fase inicial desde la esfera de la producción del conocimiento.

Si bien aún no se puede afirmar que el volumen de la producción científica argentina sobre COVID-19 es elevado o la tendencia al aumento, dado el corto período de análisis, la relevancia de este estudio está claramente apoyada por los resultados actuales, que evidencian que sí existen investigaciones argentinas publicadas caracterizada por patrón de producción de volumen considerable con colaboración científica nacional e internacional y liderazgo científico.

Los resultados obtenidos podrían interpretarse como una respuesta efectiva ante la crisis sanitaria y social sin precedentes que impuso el COVID-19, orientadas a la resolución de problemas locales, y particularmente contribuir desde la ciencia a mitigar su propagación e impacto en el sistema sanitario.

Estos resultados proporcionan a los decisores sobre la base de la evidencia datos útiles para desarrollar futuras líneas de investigación y/o potenciarlas, dado la emergencia del tema.

\section{Limitaciones de responsabilidad:}

El autor incluido en el manuscrito ha visto y aprobado la presentación de esta versión del manuscrito y asume la plena responsabilidad por su contenido.

\section{Fuentes de apoyo:}

Sin financiación externa.

\section{Originalidad:}

El presente trabajo no ha sido publicado en forma total o en parte, ni enviado a otra revista o medio para su publicación o evaluación.

Cesión de derechos:

El autor cede el derecho de autor a la Universidad Nacional de Córdoba para publicar en la RFCM y realizar la traducción en inglés.

\section{Conflicto de interés:}

El autor declara que no tener conflicto de interés relevante a este manuscrito para divulgar.

\section{Bibliografía}

1. Chahrour M, Assi S, Bejjani M, Nasrallah AA, Salhab H, Fares M, Khachfe HH. A Bibliometric Analysis of COVID-19 Research Activity: A Call for Increased Output. Cureus. 2020 Mar 21;12(3):e7357. doi: 10.7759/cureus. 7357.
2. Ministerio de Salud de la Nación. Informe diario COVID-19 Marzo 2020 [Internet]. Argentina.gob.ar. 2020 [citado 20 de junio de 2020]. Disponible en: https://www.argentina.gob.ar/coronavirus/informe-diario/marzo2020

3. Presidencia de la Nación. Aislamiento Social Preventivo y Obligatorio - Decreto 297/2020 [Internet]. Boletín Oficial de la República Argentina. 2020.2 Disponible en: https://www.boletinoficial.gob.ar/detalleAviso/primera/227042

4. Lou J, Tian SJ, Niu SM, Kang XQ, Lian HX, Zhang LX, Zhang JJ. Coronavirus disease 2019: a bibliometric analysis and review. Eur Rev Med Pharmacol Sci. 2020 Mar;24(6):3411-3421. doi: 10.26355/eurrev_202003_20712.

5. O’Brien N, Barboza-Palomino M, Ventura-León J, CaychoRodríguez T, Sandoval-Díaz JS, López-López W, Salas G. Nuevo coronavirus (COVID-19). Un análisis bibliométrico. Revista Chilena de Anestesia. 2020;49(3):408-415.

6. Zyoud SH, Al-Jabi SW. Mapping the situation of research on coronavirus disease-19 (COVID-19): a preliminary bibliometric analysis during the early stage of the outbreak. BMC Infect Dis. 2020 Aug 1;20(1):561. doi: 10.1186/s12879-020-05293-z.

7. Gregorio-Chaviano O, Limaymanta CH, López-Mesa EK. Bibliometric evaluation of Latin American contributions on COVID19. Biomedica. 2020 Oct 30;40(Supl. 2):104-115. English, Spanish. doi: 10.7705/biomedica.5571.

8. Mongeon P, Paul-Hus A. The journal coverage of Web of Science and Scopus: a comparative analysis. Scientometrics. 2016;106(1):213-28.

9. Fan J, Gao Y, Zhao N, Dai R, Zhang H, Feng X, Shi G, Tian J, Chen C, Hambly BD, Bao S. Bibliometric Analysis on COVID-19: A Comparison of Research Between English and Chinese Studies. Front Public Health. 2020 Aug 14;8:477. doi: 10.3389/fpubh.2020.00477.

10. Juárez RP, Barrere RM. Estudio Bibliométrico de la Producción Científica Argentina en Cáncer a través de las Bases de Datos Medline y Lilacs. Rev Salud Pública (Córdoba). 2017 Sep 21(2):3142. doi: 10.31052/1853.1180.v21.n2.17104.

11. Miguel S, González C, Chinchilla-Rodríguez Z. Lo local y lo global en la producción científica argentina con visibilidad en Scopus, 2008-2012. Dimensiones nacionales e internacionales de la investigación. Información, cultura y sociedad. 2015 Jun (32):59-78.

12. Zacca-González G. Producción científica latinoamericana en Salud Pública: Cuba en el contexto regional, Scopus 2003-2011 [Tesis Doctoral]. [Granada]: Universidad de Granada; 2016 [citado 20 de septiembre de 2020] Disponible en: http://hdl.handle.net/10481/40902

13. Ministerio de Ciencia, Tecnología e Innovación. Inversión en I+D [Internet]. Argentina.gob.ar. 2019 [citado 19 de septiembre de 2020]. Disponible

https://www.argentina.gob.ar/ciencia/indicadorescti/inversion

14. Cátedra Libre Ciencia, Política y Sociedad. Ciencia y tecnología en la provincia de Buenos Aires: Capacidades y propuestas. Ciencia, tecnología y política. 2020;3(4):034. doi: 10.24215/26183188e034.

15. Agencia de Promoción de la Investigación, el Desarrollo y la Innovación. COVID-19 - Convocatoria Extraordinaria [Internet]. Agencia de Promoción de la Investigación, el Desarrollo y la Innovación. $2020 . \quad$ Disponible en: http://www.agencia.mincyt.gob.ar/frontend/agencia/convocatoria/437

16. Scimago Institutions Rankings. Research and Innovation Rankings - Argentina 2020 [Internet]. Scimago Institutions Rankings. $2020 . \quad$ Disponible en: https://www.scimagoir.com/rankings.php?country=ARG 